\title{
Successful Management of Giant Placental Chorangioma by Microcoil Embolization
}

\author{
${ }^{1}$ Department of Obstetrics, Gynecology and Reproductive Sciences, \\ Magee-Womens Hospital, University of Pittsburgh Medical Center, \\ Pittsburgh, Pennsylvania \\ 2 Department of Interventional Radiology, University of Pittsburgh \\ School of Medicine, Pittsburgh, Pennsylvania \\ ${ }^{3}$ Department of Pathology, University of Pittsburgh School of \\ Medicine, Pittsburgh, Pennsylvania
}

Stephen P. Emery, MD ${ }^{1}$ Philip D. Orons, DO ${ }^{2} \quad$ Jeffrey F. Bonadio, MD ${ }^{3}$

Am J Perinatol Rep 2018;8:e230-e233.

\begin{abstract}
Address for correspondence Stephen P. Emery, MD, Department of Obstetrics, Gynecology and Reproductive Sciences, Magee-Womens Hospital, University of Pittsburgh Medical Center, 300 Halket Street, Room 2326, Pittsburgh, PA 15213 (e-mail: semery@mail.magee.edu).
\end{abstract}

Optimal prenatal management of giant placental chorangioma (also known as chorioangioma, angiomyxoma, fibroangiomyxoma, or fibroma) has yet to be determined. Interventions intended to devascularize the tumor such as interstitial laser, bipolar coagulation, fetoscopic laser photocoagulation, and chemical embolization have met mixed results. We report a minimally invasive, extra-amniotic approach, technically similar to cordocentesis, of microcoil embolization of the feeding vessel. These percutaneously placed microcoils initiate clot formation at the site of insertion and are unable to migrate through the tumor, thereby minimizing fetal harm by downstream embolic phenomena. Intervention at 26 and 22 weeks resulted in intraoperative fetal loss in the former and vaginal delivery at term of a healthy neonate in the latter. Preoperative, intraoperative, and placental findings are highlighted. The ease and safety of this procedure may alter the risk-benefit equation toward earlier intervention with potentially better clinical outcomes.

\section{Case 1}

A 27-year-old G3 P0020 was referred to our center at $25^{6 /}$ ${ }^{7}$ weeks for a suspected placental chorangioma. Ultrasound confirmed a structurally normal fetus with biometry consistent with $23^{4 / 7}$ weeks, 2 weeks less than established dates. The estimated fetal weight was $622 \mathrm{~g}$, less than the fifth percentile. The amniotic fluid index was normal at $142 \mathrm{~mm}$. Pleural fluid and ascites were identified as was cardiomegaly (70\% of chest circumference) and bilateral atrioventricular valve regurgitation. A $112 \times 84 \times 108 \mathrm{~mm}$ vascular, mixed-echogenic mass was seen with a feeding vessel, originating from the placental cord insertion of an anterior placenta, in immediate proximity to the mass consistent with a giant chorangioma. A multidisciplinary conference was convened, and the decision was made to offer intervention since no other option, such as delivery or expectant management, would likely result in a favorable outcome. Because of the location of the anterior placenta and the tumor, the feeding vessel could not be approached fetoscopically. Because of the diameter of the feeding vessel $(9 \mathrm{~mm})$, interstitial laser was felt to be likely insufficient, could result in uncontrollable hemorrhage, and could jeopardize adjacent structures. The possibility of percutaneous transuterine embolization was advanced, as the riskbenefit equation favored minimizing maternal risk given the overall poor condition of the fetus. Pathology was consulted to better understand the angioarchitecture of the tumor. Interventional radiology was consulted regarding embolization options. Given that chorangiomas may contain arteriovenous anastomoses, coil embolization was chosen over liquid techniques or microspheres as appropriately sized coils would result in complete occlusion of the feeding vessel with no risk received

March 11, 2018

accepted after revision

July 27, 2018
DOI https://doi.org/

10.1055/s-0038-1669944. ISSN 2157-6998.
Copyright $\odot 2018$ by Thieme Medical

Publishers, Inc., 333 Seventh Avenue, New York, NY 10001, USA. Tel: +1(212) 584-4662.
License terms

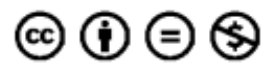


of migration through the tumor and back into the fetal circulation. A comprehensive consent process was executed that included discussion of the innovative nature of the proposed intervention. The patient expressed a desire to attempt coil embolization in conjunction with interventional radiology followed by intrauterine transfusion for anticipated severe fetal anemia. The single feeding vessel was catheterized with a 21-gauge, 15-cm needle with diamond-shaped stylet (Boston Scientific, Marlborough, MA), technically identical to intravascular access for intrauterine transfusion. A blood specimen was collected for complete blood count (CBC) and HemoCue, the latter returning at $3.0 \mathrm{mg} / \mathrm{dL}$. Two $6 \times 2 \mathrm{~mm}$ Tornado microcoils (Cook Medical, Bloomington, IN) were deployed into the feeding vessel under direct ultrasound guidance within seconds of intravascular access. Flow within the tumor ceased immediately by intraoperative color Doppler interrogation. An additional $6 \times 2 \mathrm{~mm}$ microcoil was passed as the needle was withdrawn to occlude the needle track. Unfortunately, the fetus did not survive the procedure. Nonetheless, the ease and effectiveness of the procedure, along with a very favorable maternal risk profile (compared with other alternatives), were encouraging. The case was presented at the 34th Annual Meeting of the International Fetal Medicine and Surgery Society in 2015 as a potential alternative to fetoscopic or other thermal ablative techniques.

\section{Case 2}

A 27-year-old G1 P0 was referred to our center at $22^{3 / 7}$ weeks for a suspected giant placental chorangioma. Ultrasound found an appropriately grown and structurally normal fetus but with scalp edema, pleural effusion, and ascites consistent with nonimmune hydrops. The amniotic fluid index was $65 \mathrm{~mm}$. Middle cerebral artery Doppler was indicative of severe fetal anemia. There was a $92 \times 79 \times 87-\mathrm{mm}$ hypoechoic, circumscribed vascular mass with a $6-\mathrm{mm}$ feeding vessel originating from the cord insertion of an anterior placenta and traversing the surface of the tumor before diving into the mass consistent with a giant chorangioma. A multidisciplinary conference was convened which recommended offering intervention, and patient consent was obtained for amnioinfusion, intrauterine transfusion, and coil embolization including a discussion of the innovative nature of the proposed intervention. The procedure was begun with amnioinfusion of $1,000 \mathrm{~mL}$ of warmed saline through a 22-gauge spinal needle to improve ultrasound visibility and to better define intrauterine anatomy. The intrahepatic portion of the umbilical vein was then cannulated with the same needle. A pretransfusion blood specimen was collected for $\mathrm{CBC}$ and HemoCue, the latter returning at $8 \mathrm{mg} / \mathrm{dL}$; $25 \mathrm{~mL}$ of packed red cells were transfused for a posttransfusion $\mathrm{Hgb}$ value of $15 \mathrm{mg} / \mathrm{dL}$. The needle was withdrawn. The fetal heart rate remained stable without evidence of deterioration.

After a period of observation, the decision was made to proceed with coil embolization in conjunction with interventional radiology. A 21-gauge diamond-shaped stylet needle was passed under continuous ultrasound guidance into the single feeding vessel as it entered the tumor ( $\mathbf{- F i g}$. 1). Two $6 \mathrm{~mm} \times 2 \mathrm{~mm}$ Tornado microcoils were passed into the feed-

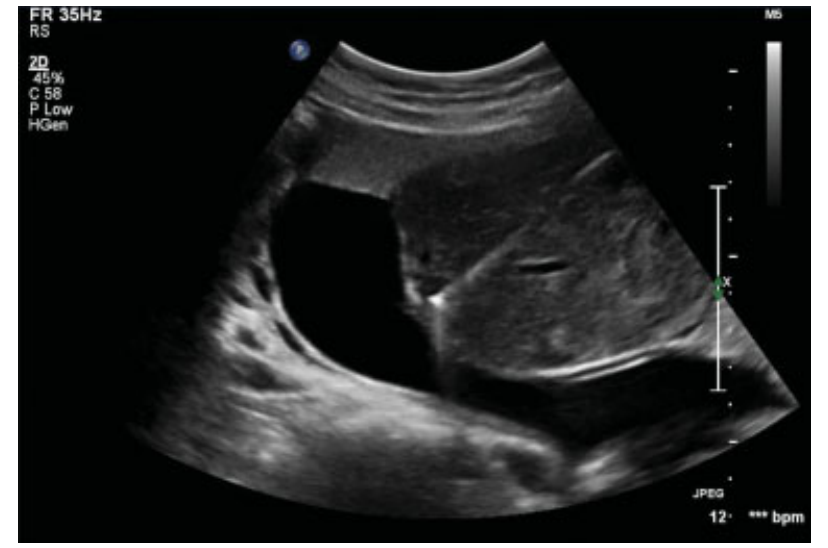

Fig. 1 Ultrasound image of 21-gauge needle placed into feeder vessel of giant placental chorioangioma. Two microcoils were then placed into the feeder vessel.

ing vessel under direct ultrasound guidance for immediate cessation of flow within the tumor by color Doppler interrogation. The needle was withdrawn without evidence of extravasation of blood into the tumor or extra-amniotic space. Ultrasound on postoperative day 1 found a viable fetus, the coils in place without migration, and no evidence of flow within the tumor. The patient underwent an otherwise routine prenatal course and delivered vaginally at term after spontaneous labor for a 2,861-g healthy infant. The placenta was fixed in formalin and transferred to our pathology department. Specimen radiograph demonstrated two Tornado coils at the site of insertion within the feeding vessel of the tumor (-Figs. 2a and 3a). Histology found a well circumscribed, completely necrotic tumor with minimal collateral effect on adjacent placental parenchyma (-Figs. $2 \mathbf{b}$ and $\mathbf{3 b}$ ).

\section{Discussion}

The optimal management of giant placental chorangioma remains undetermined. Both natural history and pathophysiology are only partially described. ${ }^{1,2}$ Maternal and fetal complications increase once the tumor exceeds 4 to $5 \mathrm{~cm}^{3}$ Maternal complications include symptomatic polyhydramnios, preterm premature rupture of membranes, preeclampsia, and mirror syndrome. Fetal loss appears to be primarily from shunt physiology and high-output failure, compounded by the secondary effect of severe anemia from microangiopathic hemolysis and red cell sequestration within the tumor. ${ }^{4}$ A third mechanism may diminish oxygen and nutrient delivery to the developing fetus due to preferential blood flow into the low-pressure vascular circuit of the tumor and away from the intervillous space (steal phenomenon). ${ }^{2}$ Finally, there is likely to be significant competitive substrate consumption by the enlarging tumor.

Although temporizing interventions such as amnioreduction for symptomatic polyhydramnios and intrauterine transfusion for severe fetal anemia may have a role in management, the primary goal of treatment is to address the underlying pathophysiology by disrupting the tumor's blood supply, thereby arresting shunt physiology, hemolysis, 

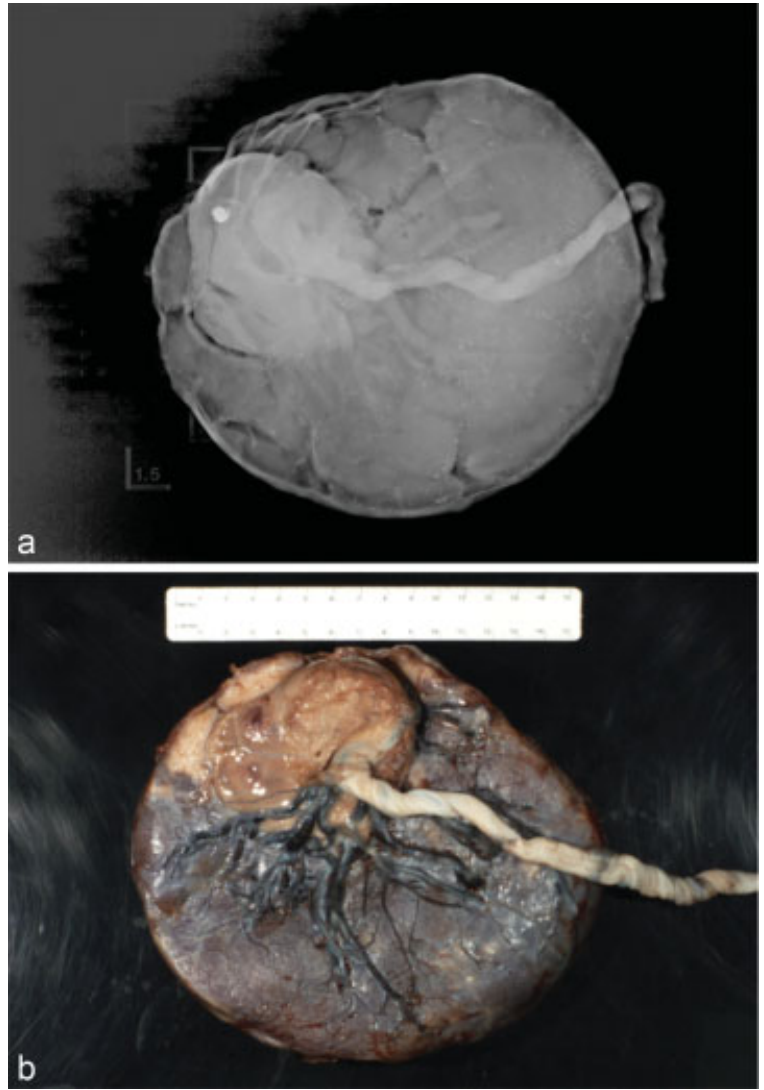

Fig. 2 (a) Specimen radiograph showing the microcoils where they were placed within the chorangioma. (b) Macroscopic appearance of fetal surface of placenta. The chorangioma is eccentrically located near the insertion of the umbilical cord and occupies $\sim 20$ to $25 \%$ of the placental disc. sequestration, steal, and substrate consumption. Tumor devascularization has been attempted using various forms of thermal energy such as bipolar cautery, interstitial laser, radiofrequency ablation, and fetoscopic laser photocoagulation, alone or in combination, each with their advantages and disadvantages. ${ }^{4-7}$ Vascular clips have been placed under fetoscopic guidance. ${ }^{7}$ Embolization has been performed with liquid agents such as ethanol, cyanoacrylate, and enbucrilate. ${ }^{8-10}$ Chorangiomas, however, may contain arteriovenous anastomoses, which can allow for passage of liquid or small particulate embolic agents through the tumor and back to the fetus. Voon et al recently reported a case of portal vein thrombosis in a newborn after use of the liquid embolic agent enbucrilate and advised caution with this material. ${ }^{10}$ This risk is overcome by the use of microcoils, which cause vascular occlusion at the point of insertion and cannot pass through the capillary bed of the tumor. ${ }^{11}$

Advantages of embolization are the ease and familiarity of the technique (technically similar to vascular access for percutaneous umbilical blood sampling/intrauterine transfusion [PUBS/IUT]), its minimally invasive nature (percutaneous, ultrasound guidance, 21-gauge needle, intravenous sedation and local anesthetic, and extra-amniotic instrumentation in the case of an anterior placenta), and lack of collateral damage that can occur with thermal energy.

Lau et al describe the use of microcoils in the management of a $10-\mathrm{cm}$ giant chorangioma with a $7.4-\mathrm{mm}$ feeding vessel using multiple (10) coils ranging in size from 2 to $6 \mathrm{~mm}$ injected into secondary branches of the feeding vessel that did not cause complete occlusion. Another nine were inserted 1 week later under general anesthesia, again with
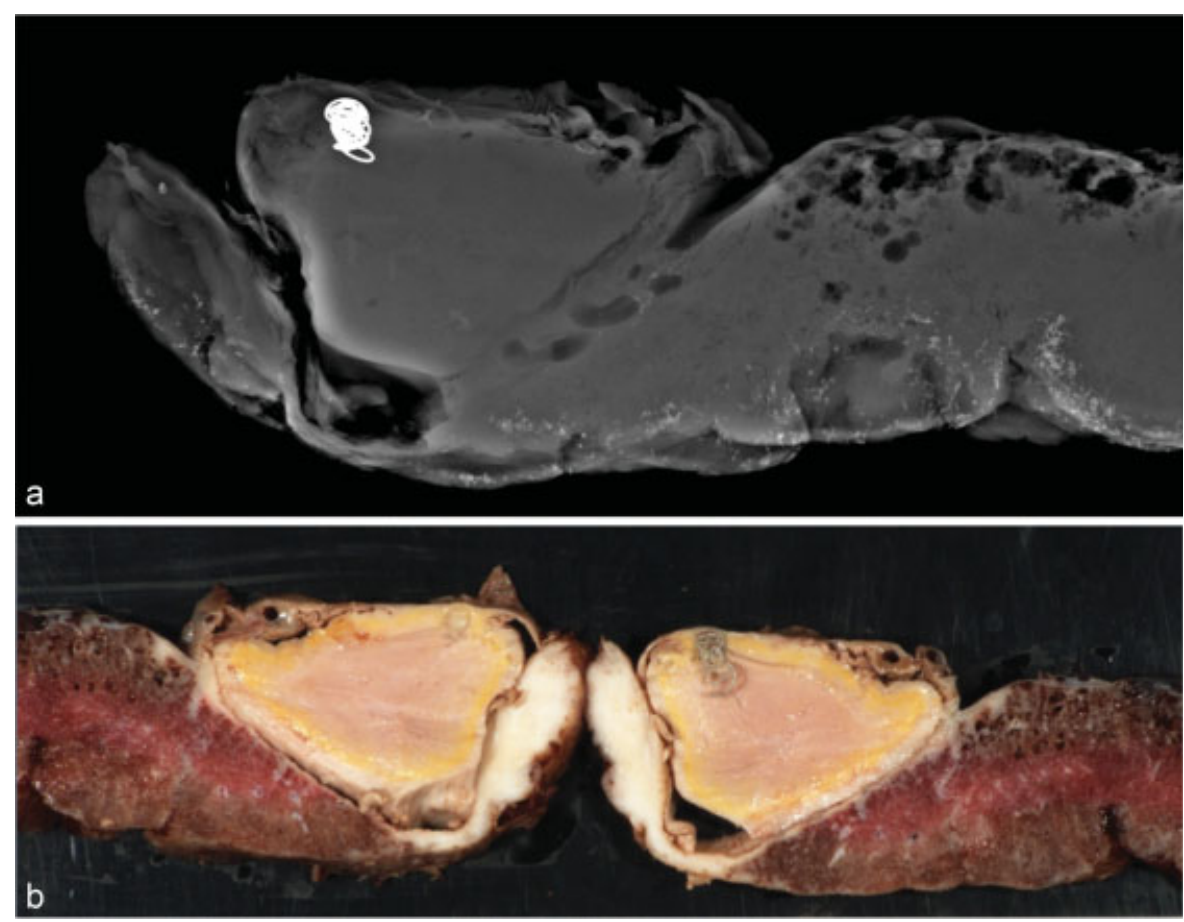

Fig. 3 (a) A specimen radiograph revealed and identified the position of two coils within the feeder vessel of the chorangioma. (b) Sectioned placental parenchyma revealed the location, color, consistency, and well-circumscribed nature of the chorangioma postcoiling, as well as the position of the two coils. 
incomplete occlusion. In contrast, we inserted two $6 \times 2 \mathrm{~mm}$ Tornado microcoils within the 6 - $\mathrm{mm}$ lumen of primary feeding vessel which resulted in rapid and complete occlusion. Location, size, and number of coils were left to the discretion of the interventional radiologist who placed the coils after intravascular access was achieved by the fetal interventionalist. We suspect that preoperative and intraoperative interdisciplinary collaborations were instrumental in our clinical success.

Due to the rare nature of giant placental chorangioma, a rigorous evaluation of natural history and response to treatment will likely require a multicenter registry, which could be accomplished through the North American Fetal Therapy Network. If coil embolization appears to be easier and safer than other techniques, the risk-benefit assessment may shift toward earlier intervention before the fetus demonstrates evidence of frank decompensation (cardiomegaly, growth restriction, oligohydramnios, severe anemia, and hydrops fetalis), which may improve clinical outcomes. In retrospect, we believe our first case was technically successful, but that the fetus was too ill to survive the hemodynamic changes that would occur with any occlusive procedure. An alternative approach could have been temporizing intrauterine transfusion as a form of intrauterine resuscitation followed by definitive embolization therapy. Maternal risks from embolization, the other half of the risk-benefit equation, were no greater than with intrauterine transfusion, which is probably the safest procedure performed in fetal intervention. Finally, with an anterior, lateral, or fundal placenta, this technique has the potential of being entirely extra-amniotic, thereby eliminating the risk of iatrogenic amniorrhexis and its deleterious consequences, chiefly preterm birth.

We applied what we had learned from our first case to the second, namely, preoperative and intraoperative interdisciplinary collaborations; amnioinfusion to better elucidate the anatomical relationships among the tumor, placenta, and feeding vessel; and intrauterine transfusion to restore intravascular volume and oxygen carrying capacity before proceeding with embolization. Also, the fetus in the second case was likely not as chronically ill as the first and was, therefore, better able to tolerate the intervention, supporting the argument that earlier intervention may result in improved pregnancy outcomes. Although it is true that our second case was not entirely extra-amniotic in that membranes were punctured for amnioinfusion and intrauterine transfusion, earlier intervention may eliminate the need for these adjuncts, and the membranes were punctured with a 22-gauge needle.
In summary, we describe a technically simple, minimally invasive, percutaneous, ultrasound-guided, extra-amniotic technique of coil embolization of a giant placental chorangioma that safely and completely occluded the feeding vessel, did not migrate back into the fetal circulation, did not cause collateral thermal damage, and exposed the mother to minimal risk. We hope that, through collaborative multicenter research, we can optimize pregnancy outcomes complicated by giant chorangioma in a scientifically rigorous and ethically sound manner. ${ }^{12}$

\section{Conflict of Interest}

None.

\section{References}

1 Fan M, Skupski DW. Placental chorioangioma: literature review. J Perinat Med 2014;42(03):273-279

2 Wu Z, Hu W. Clinical analysis of 26 patients with histologically proven placental chorioangiomas. Eur J Obstet Gynecol Reprod Biol 2016;199:156-163

3 Sepulveda W, Alcalde JL, Schnapp C, Bravo M. Perinatal outcome after prenatal diagnosis of placental chorioangioma. Obstet Gynecol 2003;102(5 Pt 1):1028-1033

4 Hosseinzadeh P, Shamshirsaz AA, Javadian P, et al. Prenatal therapy of large placental chorioangiomas: case report and review of the literature. AJP Rep 2015;5(02):e196-e202

5 Papaioannou GK, Evangelinakis N, Kourtis P, Konstantinidou A, Papantoniou N. Giant chorioangioma treated with interstitial laser coagulation. Ultrasound Obstet Gynecol 2017

6 Jones K, Tierney K, Grubbs BH, Pruetz JD, Detterich J, Chmait RH. Fetoscopic laser photocoagulation of feeding vessels to a large placental chorioangioma following fetal deterioration after amnioreduction. Fetal Diagn Ther 2012;31(03):191-195

7 Lim FY, Coleman A, Polzin W, et al. Giant chorioangiomas: perinatal outcomes and techniques in fetoscopic devascularization. Fetal Diagn Ther 2015;37(01):18-23

8 Nicolini U, Zuliani G, Caravelli E, Fogliani R, Poblete A, Roberts A. Alcohol injection: a new method of treating placental chorioangiomas. Lancet 1999;353(9165):1674-1675

9 Cheng YK, Yu SC, So PL, Leung TY. Ultrasound-guided percutaneous embolisation of placental chorioangioma using cyanoacrylate. Fetal Diagn Ther 2017;41(01):76-79

10 Voon HY, Amin R, Kok JL, Tan KS. Call for caution: neonatal portal vein thrombosis following enbucrilate embolization of placental chorioangioma. Fetal Diagn Ther 2018;43(01):77-80

11 Lau TK, Leung TY, Yu SC, To KF, Leung TN. Prenatal treatment of chorioangioma by microcoil embolisation. BJOG 2003;110(01): 70-73

12 Luks FI, Johnson A, Polzin WJ; North American Fetal Therapy Network. Innovation in maternal-fetal therapy: a position statement from the North American Fetal Therapy Network. Obstet Gynecol 2015;125(03):649-652 